VIE SCIENTIFIQUE

\title{
UN RENOUVELLEMENT DE LA COLLECTION GARNIER-FLAMMARION
}

Dans la modeste collection Garnier-Flammarion ont paru depuis 1991 de nouveaux essais de traduction de dialogues platoniciens qui, en effet, par le choix des spécialistes qualifiés, par la qualité de leur travail et des annotations qui le complètent, par l'apport aussi de divers index, témoignent du désir d'élever la collection à un niveau plus soigné et plus rigoureux, capable de satisfaire des lecteurs plus exigeants. Il est donc temps d'attirer l'attention sur cet important renouvellement qui risque d'échapper, par trop de discrétion.

Voici la liste de ces traductions de dialogues de Platon publiées entre 1991 et 1994, liste que rien n'oblige à considérer comme définitivement close ; probablement l'éditeur souhaite-t-il faire passer à la hauteur des nouveaux titres déjà publiés la totalité de sa collection platonicienne. Il est annoncé un Parménide.

L'ordre suivi ci-dessous est celui, approximatif, de la chronologie de Platon, non celui des dates de publication des nouvelles traductions. Entre parenthèses, le nom du traducteur.

Euthydème, Ion, Gorgias, Ménon (tous ces textes ont été traduits par Monique Canto-Sperber), Phédon (Monique Dixsaut), Phèdre (Luc Brisson, avec en appendice la réédition d'un texte de Jacques Derrida), Théétète (Michel Narcy), Le Sophiste (Nestor Cordero), Timée-Critias (un seul volume, Luc Brisson). Les Lettres attribuées à Platon ont été aussi traduites par Luc Brisson.

Deux remarques pour conclure ce bref aperçu. D'abord, le renouvellement entrepris pour les traductions de Platon semble bien s'étendre à celles de son plus illustre disciple, si l'on en juge par les caractères que manifestent Les Politiques (trad. Pierre Pellegrin) et le traité De l'âme (trad. Richard Bodéüs) d'Aristote : ces versions françaises dénotent le même état d'esprit que les nouvelles versions françaises de Platon. D'autre part, l'ancienne version d'E. Chambry couvrant tous les titres de Platon, à l'exception de La République, dont Chambry avait assumé l'édition et la traduction dans la «Collection Budé », aux Belles Lettres, il restait à trouver pour ce dernier dialogue un auteur qualifié : la chance voulut que le regretté Robert Baccou, prêtre à l'immense culture qui ne se limitait pas, ce qui serait déjà beaucoup, à l'histoire des sciences, préparait une thèse sur La République et se vit confier la tâche de traduire ce texte; on sait que, contrairement à d'autres, mieux protégés par l'Église catholique, le malheureux religieux succomba aux privations vers la fin de la Seconde Guerre mondiale, mais il avait eu le temps de faire passer dans les notes de sa traduction la substance de sa thèse, ce qui confère par avance à son vieil ouvrage une valeur analogue aux récents ouvrages de la collection et telle qu'il mériterait de survivre, peut-être moyennant des mises à jour, mais en sauvegardant l'ensemble.

Jean BerNHARDT.

Revue de synthèse : IV S. Nos 1-2, janv.-juin 1994. 